\title{
The Rule of Two: A Star Wars Edict or a Method of Reproducibility and Quality?
}

\begin{abstract}
Authors: Steven J. Forrester ${ }^{1}$
Affiliations: ${ }^{1}$ Division of Cardiology, Emory University School of Medicine, Atlanta, GA *Correspondence to: steven.forrester@emory.edu

Abstract

In recent years, biomedical research has faced increased scrutiny over issues related to reproducibility and quality in scientific findings(1-3). In response to this scrutiny, funding institutions and journals have implemented top-down policies for grant and manuscript review. While a positive step forward, the long-term merit of these policies is questionable given their emphasis on completing a check-list of items instead of a fundamental re-assessment of how scientific investigation is conducted. Moreover, the top-down style of management used to institute these policies can be argued as being ineffective in engaging the scientific workforce to act upon these issues. To meet current and future biomedical needs, new investigative methods that emphasize collective-thinking, teamwork, shared knowledge and cultivate change from the bottom-up are warranted. Here, a perspective on a new approach to biomedical investigation within the individual laboratory that emphasizes collaboration and quality is discussed.
\end{abstract}

Keywords: Reproducibility, quality, research integrity, universities, methods, science policy, rigor

\section{The Old Rule of Two}

There are always two. A master and apprentice. For every graduate student, postdoc or research scientist (apprentice), there is one principal investigator (PI, master) who oversees project direction and the allocation of resources and skill. When the apprentice gains sufficient experience, they leave to start their own laboratory. Or if they are a sith, they destroy their master. Luckily, science does not follow the latter edict. However, like sith philosophy, the use of this methodology in scientific investigation promotes isolation, the hoarding of knowledge and single-minded solutions to complex problems, which greatly influence the quality of biomedical discoveries. 
As the force calls to young jedi, so too does science call to scientists. Importantly, the calls for reproducibility and quality highlight the need to re-assess our version of the rule of two to instill greater quality in our findings and promote investigative methods that are aligned with the collectivist culture needed to sustain biomedical discovery. What follows is a re-interpretation of the old rule of two that is well-suited to meet the growing needs of biomedical research.

\section{The New Rule of Two}

\section{A. Hypothesis phase}

In our current old rule of two model, projects are directed by the PI to single project leads that include postdocs, graduate students and research scientists. However, this method can be ineffective in promoting shared knowledge, collective thinking and can be subjected to extreme bias(4). To remedy these inefficiencies, the rule of two could be re-interpreted to require two project leads for any given project. From the perspective of mentorship, guidance in career development and establishing a publication track-record, project lead designations are given to postdocs and graduate students; however, any scientist can be a project lead. In team discussions (two leads and PI), individual leads present their ideas and rationale followed by team member input and discussion. Discussions during hypothesis development should focus on the bigger picture of health-relevance, potential avenues of exploration and strengths and weaknesses of hypotheses and lines of research. The main goal of the hypothesis phase will be to develop and agree on a collective hypothesis and research direction for the project.

During the hypothesis phase, leads will develop the necessary skills needed to succeed as both individual investigators and effective team members. The development of a collective hypothesis/vision encourages individual input, discussion, transparency and clarity in decision making processes — and mitigates waste resulting from differing goals. Open and honest discussion about the strengths and weaknesses of hypotheses creates an environment where concerns and opposing viewpoints are readily presented, which is a strength to this style of investigation. Moreover, continual input and feedback from multiple minds encourages shared learning and further develops the critical thinking skills of every team member. This is especially important in situations of skill and knowledge disparity between project leads such as a postdoc and beginning graduate student. By requiring — and documenting — collective decision making, experienced leads 
and PIs are encouraged to take an active role in providing mentoring and support to lessexperienced trainees. This will give less-experienced trainees the guidance they need as well as provide the more advanced lead with mentoring experience. Likewise, documentation of individual and group decision making will highlight areas of improvement for each team member.

The rule of two approach requires the PI to wear many hats including those of mentor, manager and facilitator. For bottom-up initiatives to work, management must support and champion the idea to ensure work-force buy-in and sustained action (5). PIs must bring together project leads and coach and manage team members as they develop individually and as a team. As scientists, PIs are also responsible for giving their input into the lines of research that project leads agree on. This introduces a more experienced mind into the discussion and can further strengthen the direction of the project and enhance skill development; however, it is critical that PIs encourage autonomy of project leads and not steer the direction of research themselves. For bottom-up initiatives to work, employees must feel a sense of control in the decided direction of investigation, and not feel as though their input is unwarranted or undermined. Thus, PIs must embrace the mentoring role and find ways to guide, manage, and support the research team without overstepping into the micromanagement realm. This is vitally important for less-experienced leads. These trainees may require more guidance and managing in the beginning of their training, but effort should be focused on teaching them the skills to ask and test their own questions, as opposed to instructing them on what to do. In these scenarios, working daily with an experienced project lead will promote knowledge sharing, individual development and trust between team members. The role of PI, in some ways, is the most difficult position to have in this approach, but the reward is well-worth the needed investment.

\section{B. Experimental phase}

After a collective hypothesis is agreed upon, project leads brainstorm and discuss the most prudent experiments needed to test their hypothesis. The key here is simplicity. Pursuing answers to puzzling questions can be enticing at times and often leads to the juggling of multiple experiments. In many cases, overburden and multi-tasking introduce inefficiency into work, which reduces performance and wastes resources and time $(6,7)$. However, by aligning goals towards a collective hypothesis, teams will be effective in choosing appropriate experiments and disregarding wasteful 
and time-consuming ancillary items. Agreed upon experiments should aim to test the most important aspects of a given hypothesis and should be designed with quality built-in to promote rigor and reproducibility. This can be accomplished by having project leads blind each other to their respective experiments and make use of standard practices of rigor and reproducibility $(8-10)$. In addition, conducting experiments in parallel — whether they be the same or different — promotes a culture of shared knowledge, as both leads consult and train one another in best practices. Transparency and openness are also integral to ensuring quality in scientific findings. During the experimental phase, methods of reproducibility and rigor must be documented and agreed upon by all parties (both project leads and PI). After data has been acquired, both leads will interpret, discuss and agree on the next step with PI consultation.

Like the hypothesis phase, the experimental phase encourages open discussion concerning the most pertinent experiments needed to test a given hypothesis. As a result, experiments are morelikely to be well-thought out and provide quality findings that will propel continued investigation. Furthermore, this phase builds quality into the experimental setup by requiring leads and PIs to agree on, perform and document instituted methods of reproducibility. A reduction in multitasking and an emphasis of focus on a given project by two leads will also reduce worker overburden and promote quality and efficiency in their investigation.

Randomization is integral to the sharing of knowledge in the rule of two. Often, laboratories have multiple on-going projects and start new projects after the completion of others. Upon completion of a single project, teams are divided, and project leads for a new project are randomized. Randomizing ensures no two people work together on consecutive projects and facilitates teambuilding and knowledge dissemination throughout the laboratory. It will also be incumbent upon the PI to ensure all employees rotate as project leads to promote balanced skill development (Fig 1). 


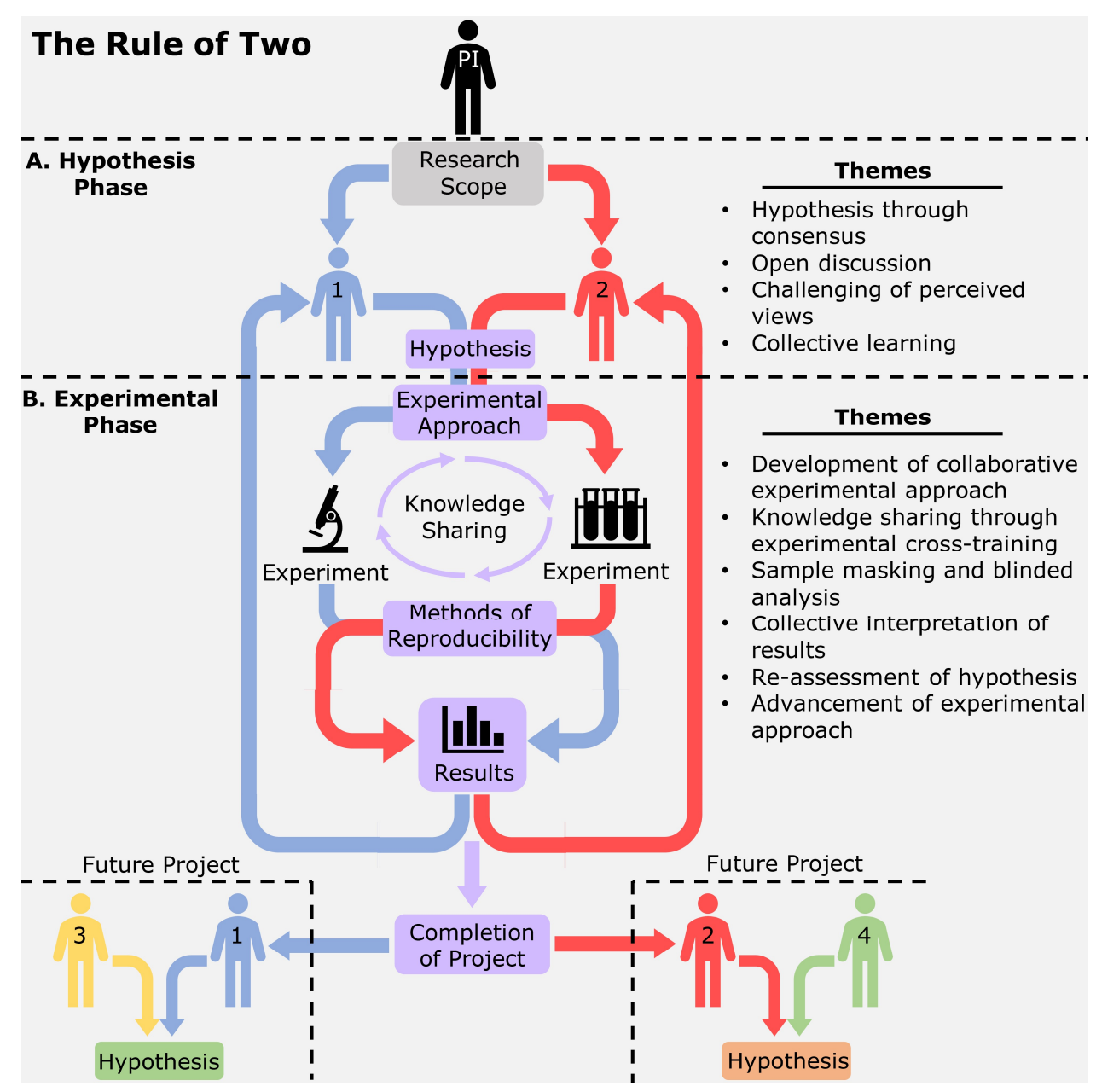

Figure 1. The rule of two. Within any given research environment, research agenda and specific hypotheses are developed through consensus between two project leads under the guidance and management of a PI. Team-based hypotheses are tested through collective experimental approaches that promote knowledge sharing, team-building, reproducibility, and quality. Upon interpretation of results, groups re-assess collective hypotheses and experimental approaches for continued investigation. At the start of new projects, team members are randomized into groups to facilitate knowledge sharing.

\section{Instituting the New Rule of Two}

The new rule of two is an investigative style that is well-suited to meet current and future biomedical needs including collective decision making, increased quality of findings, patientoriented research and teamwork. It encourages investigators to reflect on their contribution to 
scientific quality, address their subconscious biases and requires active participation in refining how biomedical research is conducted by instituting it within their own work setting. However, there will be significant hurdles in instituting this method that will require significant time and effort. Most important, the current culture of biomedical research does not favor the collectivisttype of investigation that this method promotes. Although collectivism is vital to understanding and addressing biomedical needs, individualism remains the preferred route for career advancement and security. Until the biomedical community shifts its culture away from individualism, it will be difficult to institute more collective and team-based approaches to scientific discovery. Nonetheless, through bottom-up initiatives like the rule of two, the spreading of team-oriented approaches from laboratory to laboratory will slowly change the scientific landscape far more effectively than any top-down policy.

\section{Concluding Remarks}

Overall, the new rule of two is a simple method that uses team-based problem solving to conduct scientific investigation of greater quality. In addition to its use within the individual laboratory, this method would be effective in collaborative networks and projects between investigators. Through collaboration, this method can be continually refined and spread to individual laboratories until it finally becomes a common workplace practice. Thus, it is a true bottom-up initiative that puts the power of change in the hands of the scientific workforce. With support, patience and continual improvement, the new rule of two can create the needed change in biomedical research. However, it will require effort on everyone's part to succeed. In the famous words of Yoda, "Do. Or do not. There is no try."

Acknowledgments: I'd like to thank Dr. Keisuke Kawata for his excellent insight during the preparation of this manuscript

Funding: No funding to disclose 


\section{References:}

1. M. Baker, 1,500 scientists lift the lid on reproducibility. Nature News 533, 452 (2016).

2. D. A. Eisner, Reproducibility of science: Fraud, impact factors and carelessness. Journal of Molecular and Cellular Cardiology 114, 364-368 (2018).

3. A. Casadevall, F. C. Fang, Reproducible Science. Infection and Immunity 78, 4972-4975 (2010).

4. L. Holman, M. L. Head, R. Lanfear, M. D. Jennions, Evidence of Experimental Bias in the Life Sciences: Why We Need Blind Data Recording. PLOS Biology 13, e1002190 (2015).

5. J. P. Kotter, Leading change. (Harvard business press, 2012).

6. J. S. Rubinstein, D. E. Meyer, J. E. Evans, Executive control of cognitive processes in task switching. Journal of Experimental Psychology: Human Perception and Performance 27, 763-797 (2001).

7. A. Zika-Viktorsson, P. Sundström, M. Engwall, Project overload: An exploratory study of work and management in multi-project settings. International Journal of Project Management 24, 385-394 (2006).

8. M. R. Munafò et al., A manifesto for reproducible science. Nature Human Behaviour 1, 0021 (2017).

9. C. G. Begley, J. P. A. Ioannidis, Reproducibility in Science. Improving the Standard for Basic and Preclinical Research 116, 116-126 (2015).

10. R. Bolli, New Initiatives to Improve the Rigor and Reproducibility of Articles Published in Circulation Research. Circulation Research 121, 472-479 (2017). 